\title{
Glucose sensor based on the LUQUEN principle
}

\author{
G. L. J. Hesselink, H. J. M. Kreuwel, P. V. Lambeck, H. J. v. d. Bovenkamp, \\ J. F. J. Engbersen, D. N. Reinhoudt and Th. J. A. Popma \\ MESA, Institute for Micro Electronics, Materials Engineering, Sensor \& Actuators, University of Twente, P.O. Box 217, \\ 7500 AE Enschede (Netherlands)
}

\begin{abstract}
A new type of glucose sensor based on luminescence quenching (LUQUEN) is proposed. In this LUQUEN principle the concentration induced quenching is enlarged by radiationless (excited-state) energy transfer within the luminescent material. We describe the integrated optical detection part and the chemo-optical interface, in which the concentration induced optical changes are based on the binding of glucose. Some experiments on the internal energy transfer are reported, and the results are discussed.
\end{abstract}

\section{Introduction}

There is a need for the development of a glucose sensor that can be used easily and on line for analytical purposes in chemical laboratories, food and fermentation industry, and medical applications. Instead of the well known enzymatic conversion [1], we have chosen another approach, based on the measurement of optical effects caused by reversible binding of glucose to a selective optically labeled receptor. This receptor system will function as an interface between the chemical and the optical domain. In principle, the optical response upon the glucose binding can be a change in either the absorptive, the refractive or the luminescent properties of the receptor.

If we pay first attention to the absorption a most promising method is based on quenching of luminescence emission (LUQUEN) by these receptor molecules, applied as a interface layer on top of a luminescent material. Using luminescent materials, showing a large radiationless transfer between the centres, an optical gain is achieved.

Strong points of the LUQUEN principle are the great sensitivity achieved by this gain and the small sensitivity for changes in optical properties of the chemical environment. By using waveguides, which can be used for the transport of the light to and from the luminescent material, a small versatile sensor can be obtained. It has been shown by Kreuwel [2], using a non-optimized prototype based on this principle, that detection of a fractional complexation as small as $10^{-3}$ to $10^{-5}$ of the reported molecules (in this case bromo cresol purple) is feasible. This detection limit will be strongly dependent on the extent of the energy transfer in the luminescent material and some experimental results about this phenomena will be presented and discussed in this paper. But before doing so, we will describe the sensor principle in more detail, in particular the optical detection part and the opto-chemical interface aimed for glucose detection.

\section{Optical detection}

\section{Energy transfer}

An excited luminescent molecule can return to the ground state by emission of light, but also by transferring its excitation energy (often called exciton) radiationless to another molecule. The physical origin of this exciton transfer is often an electric dipole-dipole interaction between the exciton donor and the exciton acceptor. It can be calculated [3] that the probability of this transfer is strongly dependent on the distance $R$ between both centres, involved in the transfer:

$k=\frac{1}{\tau_{0}}\left(\frac{R_{0}}{R}\right)^{6}$

where $\tau_{0}$ is the luminescent decay time of the luminescent centre, if no transfer occurred. $R_{0}$ is the critical distance, i.e. the distance for which the 
probability for transfer is equal to the probability for decay. For dipole-dipole interaction the critical distance is given by the classical Förster-Dexter expression:

$R_{0}=\left(\frac{3 h^{4} c^{4} Q}{4 \pi n^{4}} \int \frac{f_{\mathrm{D}}(E) F_{\mathrm{A}}(E)}{E^{4}} \mathrm{~d} E\right)^{-1 / 6}$

Herein $Q$ is the integrated absorption cross section, $f_{\mathrm{D}}(E)$ the normalized emission spectrum of the donor, $F_{\mathrm{A}}(E)$ the normalized absorption spectrum of the acceptor, $n$ is the refractive index of the surrounding medium. The above formula holds as well for two identical molecules as for two molecules of different type.

\section{Sensing based on luminescence quenching}

For sensing we apply principally a simple setup consisting of a layer of luminescent material with on top of it a thin interface layer consisting of receptors. It is required that complexed receptors show a quenching potential completely different from that of uncomplexed receptors. This can be achieved in such a way that the emission spectrum of the luminescent centre shows a large overlap with the absorption spectrum of the complexed receptor molecules but a small overlap (preferentially zero) with the absorption spectrum of the uncomplexed ones. Besides the receptors have to return to the ground state radiationless. By choosing a luminescent material with large internal excitation transfer, we increase the probability for an exciton entering the effective region of a complexed receptor and thus the quenching effect of that receptor. In that way the quenching centre is able to consume a lot of photons, originally captured by the luminescent centres, and presented to it as excitons. By this mechanism, comparable to antenna action, a photon consumption by a complexed receptor molecule is obtained that is much larger than can be reached by pure absorption in the absence of the luminescent material. Hence we have introduced an optical gain. The following factors will affect this gain:

(i) Exciton transfer. Increasing the internal exciton transfer in the luminescent material, e.g. by increasing the concentration, and by choosing centres with large $R_{0}$.

(ii) The quenching action of the complexed receptor. For increasing the gain we must make an effective quencher. This means matching its ab-

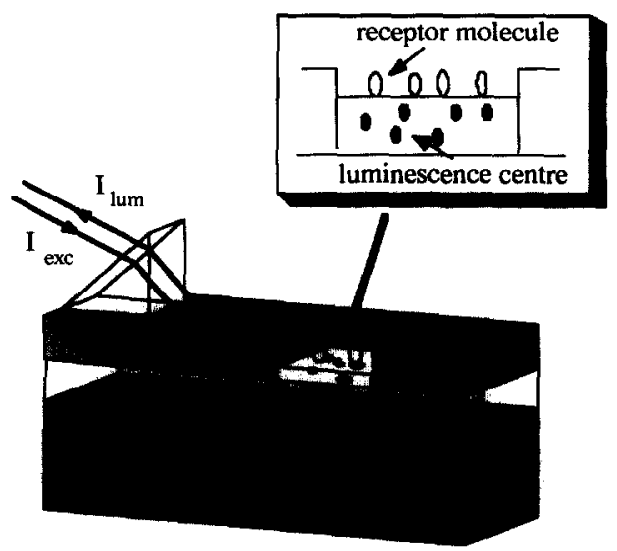

Fig. 1. Cross section of the LUQUEN sensor.

sorption spectrum to the emission of the luminescent centre and radiationless return to the ground state with a decay time small compared to the decay time of the luminescent centre. The latter causes the receptor to be almost continuously available for the excitons.

Because of the short range of energy transfer, there is only a small sensitivity for molecules outside the interface.

\section{Layout}

The system part described above can be incorporated in a waveguiding system (fiber or integrated optics) that takes care of the transport of the excitation light the emission light, taking advantage of the fact that a large part ( $\approx 60 \%$ ) of the emission light can be collected as a waveguide mode [2].

The prototype system is depicted in Fig. 1. For the waveguide we used a SiON deposited on top of a $\mathrm{SiO}_{2}$ layer, and partly screened from the environment by a $\mathrm{SiO}_{2}$ cladding layer. In the part without cladding a $1 \mu \mathrm{m}$ thick luminescent layer is spincoated which consists of polyurethane (Neorenz) with luminescent Rhodamine-6G incorporated to a typical concentration of $6 \times 10^{-4} \mathrm{Mol}$. The Rhodamine is excited with green light (typically $475 \mathrm{~nm}$ ) and gives a red emission (typically $600 \mathrm{~nm}$ ). The interface layer on top of it is described in the next section.

\section{Interface}

For the receptor we use a glucose-binding protein (GPB). In the first stage we will use the 
commercially available molecule concanavaline A (Con A) showing an association constant of about $4 \times 10^{2} \mathrm{~mol}^{-1}$. Later on proteins with larger specificity and larger association constants will be used. Because the glucose binding of Con A does not introduce absorption changes in the visible, a reporting function has to be added. Several reporting schemes are possible. A first option is a competition between glucose and the reporting group (the latter being covalently bound to the protein) at the binding site of the receptor molecule; binding of glucose will result in a variation of the microenvironment of the reporter molecule. This can be signalled by choosing strongly solvatochromic dyes, for example the merocyanines, spirans and the pyridinium- $N$-phenolate betaines, the solvatochromic properties of which have been reported elsewhere [4]. A shift in the absorption band, corresponding with our demands, is feasible. The protein can be immobilized on the polyurethane by covalent binding [5].

\section{Measurements}

The most essential point in this sensor is the energy transfer. Energy transfer in the luminescent material can be measured in different ways. By using the method of concentration quenching of fluorescence polarization [6], information on the critical distance $R_{0}$ between the luminescent centres can be obtained. By measuring the luminescence quenching obtained in a system as presented in Fig. 1, the excitation diffussion length can be calculated from the dependency of the quenching on the thickness of the layer.

\section{Polarization measurements}

If an excited molecule itself emits radiation, the polarization states of excited and emitted light are correlated. A common assumption in most theories about fluorescence depolarization is that this correlation will already be lost on an immediate exciton transfer step between two identical molecules [6]. This means that measuring the polarization of the emitted light as a function of the mean mutual distances gives information about the critical distance $R_{0}$. This is called concentration quenching of fluorescence polarization (cqfp).

The quantity to be measured is the anisotropy, defined as:
$A=\frac{I_{\|}-I_{\perp}}{I_{\|}+2 I_{\perp}}$

where $I_{\|}$and $I_{\perp}$ are the intensities of the light with polarization parallel and perpendicular, respectively, with respect to the incoming light.

From the dependency of the normalized anisotropy $A / A_{0}$ on the normalized concentration $c / c_{0}$, with $c_{0}=\left(\frac{4}{3} \pi R_{0}^{3}\right)^{-1}$, of the luminescent centres, the critical distance $R_{0}$ can be obtained by using the method given by Craver and Know [6c] (Fig. 2).

For $6 \times 10^{-4} \mathrm{M}$ Rhodamine in polyurethane we have calculated in this way $R_{0}=65 \AA$.

\section{Measurements of luminescence quenching}

Exciton concentration $\left(c^{*}\right)$ in a luminescent layer can be described by a continuity equation:

$D \nabla c^{*}(\boldsymbol{r})+\alpha P_{\mathrm{EXC}}(\boldsymbol{r})-\frac{c^{*}(\boldsymbol{r})}{\tau_{0}}=\frac{\mathrm{d} c^{*}(\boldsymbol{r})}{\mathrm{d} t}$

wherein the first term describes a diffusion, the second the excitation, and the third term the decay of the luminescent molecules in the system given in Fig. 1, in which a monomolecular acceptor layer is applied, the problem can be approximated as onedimensional. By assuming, the $P_{\text {EXC }}$ is position independent (a rather rough approximation) the steady state condition can be found from the equation

$D \frac{\mathrm{d}^{2}}{\mathrm{~d} x^{2}} c^{*}(x)+\alpha \cdot P_{\text {EXC }}-\frac{c^{*}(x)}{\tau_{0}}=0$

applying the proper boundary conditions. Assuming the probability of the energy transfer to the monolayer receptors to be much larger than the probability of decay, from this equation a simple

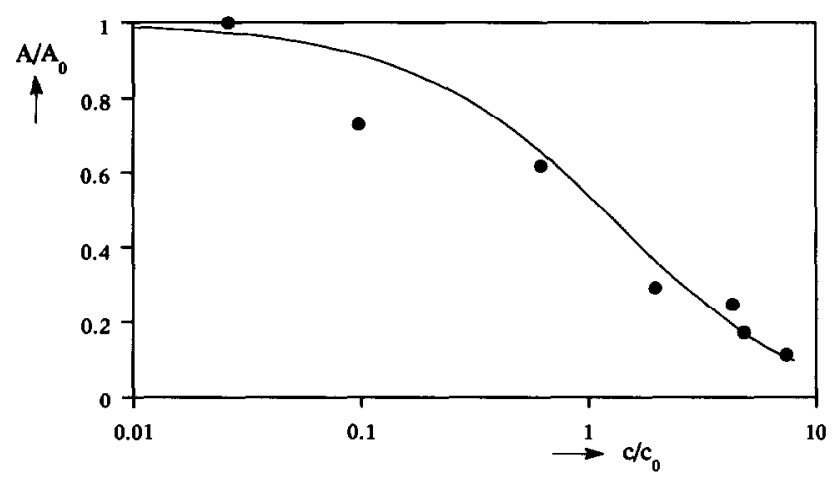

Fig. 2. Fluorescence polarization measurement with fit from Craver and Knox [6c]. 


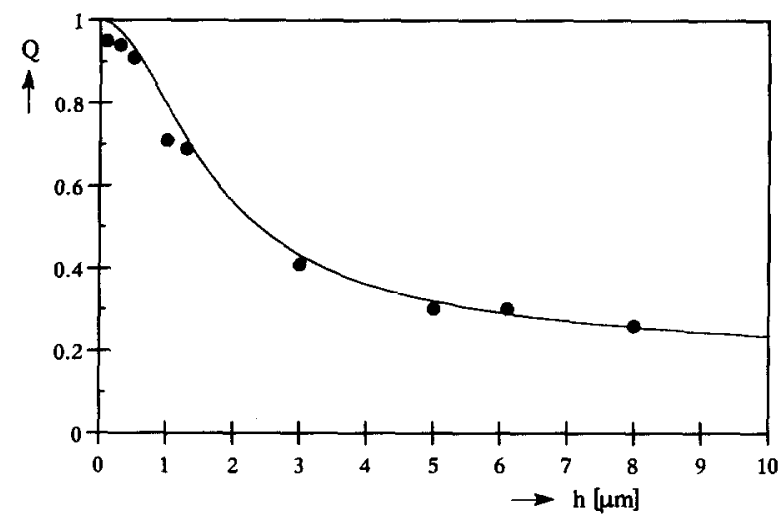

Fig. 3. Exiton diffusion length measurement and fit with eqn. (6).

expression for the quenching can be deduced [2]

$Q \approx \frac{\tanh h / d}{h / d}$

wherein $h$ is the thickness of the luminescent layer and $d$ represents the exciton diffusion length $d=\sqrt{\mathrm{D} \tau_{0}}$ which is the mean distance an exciton can travel before it decays. The quenching $Q$ is defined as $Q=1-I / I_{0}$, where $I$ is the luminescent intensity with and $I_{0}$ without quenching. Applying bromo cresol purple (BCP) in the basic state as acceptor molecules, from $Q(d)$ measurements (Fig. 3) a diffusion length of $1 \mu \mathrm{m}$ can be calculated.

\section{Discussion}

By substitution of experimentally obtained absorption and emission data of the Rhodamine in relation ( 2 ) we calculate $R_{0} \approx 76 \AA$, a value about $10 \%$ larger than the experimental one. This difference can be caused by the assumption of dipoledipole interaction or by experimental uncertainties.

The microscopic experimental data $\left(R_{0}=65 \AA\right)$ could be related to the macroscopic exciton diffu- sion length $(d=1 \mu \mathrm{m})$ by considering the mean mutual distance between the centres at the experimental concentration: $R_{\mathrm{av}}=87 \AA$. It means that an exciton should have to be transferred more than 100 times in order to diffuse over $1 \mu \mathrm{m}$. This seems to be in contradiction to the fact that for this value of the mutual distance the hopping probability would be smaller than the decay probability. By applying relations between $R_{0}$ and $d$ from Gouchanour et al. [7] this discrepancy can also be seen.

This contradiction could be explained either by assuming chains of Rhodamine molecules with smaller distances which form gateways for the exciton, or by assuming that the polyurethane matrix has a certain influence on the transfer, e.g. by affecting the mutual orientations. Further research is in progress.

\section{References}

1 Sl. Brocks, R. E. Ashby, A. P. F. Turner, M. R. Clader and D. J. Clarke, Biosensors, 3 (1987) 45.

2 H. J. M. Kreuwel, Planar waveguide sensors for the chemical domain, Ph.D. Thesis, Enschede, The Netherlands, 1988, Ch. 6.

3 Th. Förster, Zwischenmolekulare Energiewanderung und Fluoreszenz, Ann. Phys., 2 (1948) 55.

4 H. J. van de Bovenkamp, G. L. J. Hesselink, P. V. Lambeck, Th J. A. Popma, J. F. J. Engbersen and D. N. Reinhoudt, Synthesis and screening of dyes for the development of an optical glucose sensor based on a new concept, Lectines: Bio., Biochem., Clin. Biochem. (1991) to be published.

5 G. H. M. Engbers, Development of heparinized materials with an improved blood compatibility, Ph.D. Thesis, Enschede, The Netherlands, 1990.

6 (a) R. S. Knox, Theory of polarization quenching by excitation transfer, Physica, 39 (1968) 361, (b) F. W. Craver, Theory of concentration quenching of fluorescence polarization in two-dimensional solutions, Mol. Phys, 22 (1971) 403-420, (c) F. W. Craver and R. W. Knox, Theory of polarization quenching by excitation transfer, II. Anisotropy and second-neighbour considerations, $\mathrm{Mol}$. Phys., 22 (1971) 385-402.

7 C. R. Gouchanour, H. C. Anderson and M. D. Fayer, Electronic excited state transport in solution, J. Chem. Phys, 70 (1979) $4254-4270$. 